Classification

Physics Abstracts

$61.30-61.30 \mathrm{~J}-63.20$

\title{
Diffuse X-ray Scattering from Freely Suspended Strands of a Discotic Liquid Crystal
}

\author{
Patrick Davidson $\left({ }^{1}\right)$, Marianne Clerc $\left({ }^{1}\right)$, Surya S. Ghosh $\left({ }^{2}\right)$, Nicholas C. Maliszewskyj $\left({ }^{2}\right)$, \\ Paul A. Heiney $\left({ }^{2}\right)$, John Hynes Jr. $\left({ }^{3}\right)$ and Amos B. Smith, $\operatorname{III}\left({ }^{3}\right)$ \\ ( ${ }^{1}$ ) Laboratoire de Physique des Solides Associé au C.N.R.S., Université Paris-Sud, Bâtiment \\ 510, 91405 Orsay, France \\ $\left({ }^{2}\right)$ Department of Physics and Laboratory for Research on the Structure of Matter, University \\ of Pennsylvania, Philadelphia PA 19104, U.S.A. \\ $\left({ }^{3}\right)$ Department of Chemistry and Laboratory for Research on the Structure of Matter, University \\ of Pennsylvania, Philadelphia PA 19104, U.S.A.
}

(Received 17 August 1994, accepted 28 October 1994)

\begin{abstract}
We present the first quantitative measurements of diffuse scattering in the vicinity of the Bragg $\{100\}$ peaks of a hexagonal discotic liquid crystal. The scattering takes the form of a toroid lying in the $H K 0$ plane. The cross-section of the diffuse scattering in the $H O L$ plane is an ellipse elongated along the $L$ direction, in reasonable agreement with calculations for the thermal diffuse scattering. The cross section in the $H K 0$ plane has an unexpectedly smooth six-fold sinusoidal modulation.
\end{abstract}

\section{Introduction}

Discotic liquid crystals are typically composed of molecules with a disk-like core and 6-8 aliphatic tails [1]. Depending on the temperature and molecular geometry, discogenic molecules can form either discotic nematic phases $\left(\mathrm{N}_{\mathrm{D}}\right)$ or discotic columnar phases (D) with long-range ordering of the columns in a two-dimensional lattice and only short-range intracolumnar or$\operatorname{der}[2,3]$. The commonly observed hexagonal discotic $\left(D_{h}\right)$ phase consists of a hexagonal array of columns with only fluid-like intracolumnar order and consequently no long-range columncolumn correlation of the molecular heights. A rectangular discotic $\left(D_{r}\right)$ phase and other phases with lower symmetry have also been observed $[4,5]$.

Since columnar phases appear as two-dimensional assemblies of one-dimensional liquids, they are structurally intermediate between smectic phases (one-dimensional stacks of twodimensional liquids) and true crystals. The X-ray scattering by smectic phases has been extensively studied. Detailed analysis of peak lineshapes has shown that these phases do not possess true long-range positional order [6]. Furthermore, an analysis of diffuse scattering in a smectic phase can give information on its defects and elastic properties. For example, in the 
case of the smectic-A phase of a side-chain polymer [7], the observation of diffuse scattering in the shape of "butterfly wings" was interpreted as arising from edge dislocations. Comparison of the scattering to the Fourier transformation of the elastic deformation field suggested that the compression modulus of this polymeric phase was unusually large.

In contrast, columnar phases do possess true long range two-dimensional positional order, which gives rise to a set of true Bragg peaks lying on a hexagonal net in a single plane of reciprocal space. (In this paper we will refer to these as the $\{H K 0\}$ Bragg peaks and the plane in which they lie as the basal plane, spanned by the vector $\left.\mathbf{Q}_{\mathrm{b}}=Q_{x} \hat{\mathbf{x}}+Q_{y} \hat{\mathbf{y}}\right)$. The short-range ordering of the cores within the columns results in a diffuse feature centered on the $Q_{z}$ axis, which may be referred to as the 001 "peak" although it is much broader than a Bragg peak. In addition, intracolumnar correlations of the highly disordered aliphatic tails can give rise to a shell-like diffuse scattering feature, rougnly centered on the $Q_{z}$ axis but at a different magnitude of $|\mathbf{Q}|[3,8]$. Since there are no intercolumnar correlations, no $\{H K L\}$ peaks are seen.

Previous structural measurements of discotic liquid crystals have focussed on the nature and symmetry of the two-dimensional ordering of the columns, as evinced by the intensities and positions of the $\{H K 0\}$ Bragg peaks, and the nature of the intracolumnar disorder, as evinced by the shape of the 001 peak and tail-tail scattering. However, thermal fluctuations of the columns and defects in the periodic columnar order can result in additional diffuse scattering at a characteristic momentum transfer $|\mathbf{Q}| \sim 2 \pi /$ (intercolumnar distance), similar to that seen in smectics [7]. To our knowledge, the existence of diffuse scattering in a $D_{h}$ phase has been reported only once: the diffraction pattern of hexapentyloxytriphenylene displays diffuse sheets parallel to the axes of the columns and the $\{H K 0\}$ rows [9]. These diffuse sheets were presumed to arise from low frequency bending modes of the columns.

In this paper we present the first quantitative measurements of the low-angle diffuse X-ray scattering from highly oriented, freely suspended strands of a discotic liquid crystal. We find that the diffuse scattering is confined to a toroid in the basal plane of the Bragg $\{H K 0\}$ points, as shown schematically in Figure 1.

The diffuse scattering intensity is at its maximum near the Bragg $\{100\}$ points. The crosssection in the $H 0 L$ plane of this diffuse scattering toroid is elliptical, confirming the expected anisotropy between the momentum transfers in the plane of the two-dimensional lattice and along the direction of the column axis. The cross section in the $H K O$ plane is a circular ring with a simple sinusoidal modulation which reflects the six-fold symmetry of the two-dimensional lattice of the cores.

A number of different factors can give rise to diffuse scattering, in liquid crystals as well as in crystals. Our measurements are in qualitative agreement with calculations [10,11] of the diffuse scattering from "phonons" (collective thermal displacive excitations) in the liquid crystal. A similar form for the diffuse scattering can also arise from static defects in the columnar structure [13-15]. In future work [16] we will address the extent to which aliphatic tail disorder contributes to the diffuse scattering in the $(H K O)$ plane.

The remainder of this paper will proceed as follows. In Section 2 we discuss the experimental techniques used. In Section 3 we present the results of our X-ray measurements. Section 4 discusses our analysis of the shape of the diffuse scattering in reciprocal space. In Section 5 we derive the characteristic form of scattering from phonons in a discotic liquid crystal. We provide a critical comparison between our data and the phonon scattering model in Section 6 . 


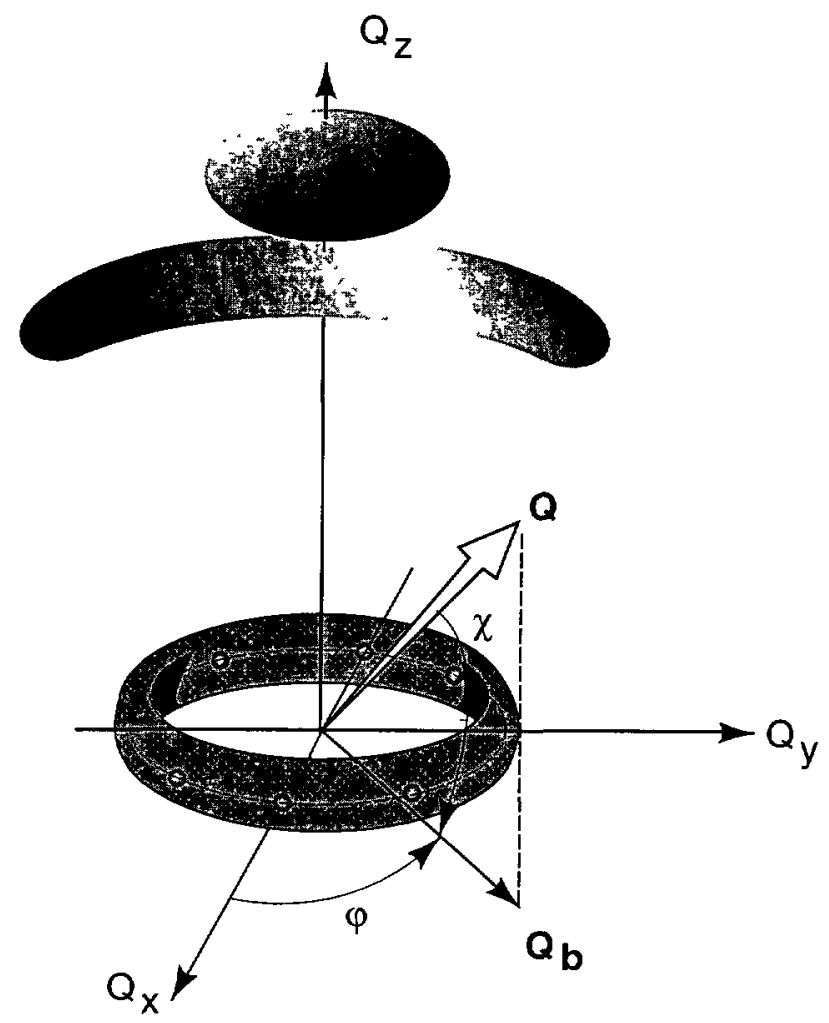

Fig. 1. - Schematic representation of reciprocal space depicting the toroid and the Bragg $\{100\}$ points in the basal plane. The broad "001" scattering region due to intracolumnar core-core correlations is found on the $Q_{z}$ axis. A shell of larger angle diffuse scattering is due to the intercolumnar tail-tail scattering. $\mathbf{Q}_{b}$ is the projection of the scattering vector $\mathbf{Q}$ on to the basal plane.

\section{Experimental}

The material studied was hexa(hexylthio)triphenylene (HHTT) (Fig. 2). This compound has the phase sequence:

$$
\mathrm{K} \underset{62^{\circ} \mathrm{C}}{\longleftrightarrow} \mathrm{H} \underset{70^{\circ} \mathrm{C}}{\stackrel{\longrightarrow}{\longleftrightarrow}} \mathrm{D}_{\mathrm{h}} \underset{93^{\circ} \mathrm{C}}{\longleftrightarrow} \mathrm{I}
$$
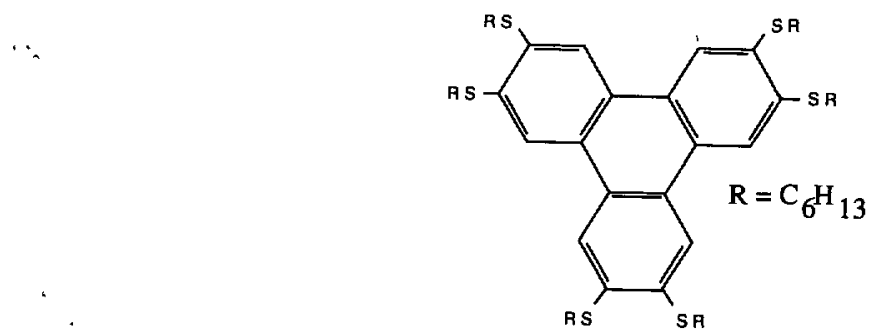

Fig. 2 - Structure of hexa(hexylthio)triphenylene (HHTT). 
where $\mathrm{K}$ is a monoclinic crystal, $\mathrm{H}$ is a helical crystalline phase, $\mathrm{D}_{\mathrm{h}}$ is a hexagonal discotic liquid crystal phase, and I is the isotropic phase [17-20]. Pure HHTT was synthesized and purified as previously described [21-23]. Freely suspended strands [24,25] of HHTT were grown [26] in a temperature-controlled environment.

Preliminary, qualitative measurements were made using a simplified strand apparatus, with $\mathrm{X}$-ray detection using photographic film [27]. For these measurements, the temperature chosen was $90^{\circ} \mathrm{C}$, within the $D_{h}$ phase. More quantitative $\mathrm{X}$-ray measurements (at $75^{\circ} \mathrm{C}$, also in the $D_{h}$ phase) were performed using an Elliott GX-13 rotating anode generator with an apparent source diameter of $100 \mu \mathrm{m}$. A vertically focussed $\operatorname{LiF}(200)$ monochromator selected the $\mathrm{Cu} \mathrm{K} \mathrm{K}_{\alpha 1}$ line, producing an incident flux of roughly $10^{7}$ photons $/ \mathrm{s}$ in a $400 \mu \mathrm{m}$ diameter spot at the sample position. The direction of the outgoing beam was determined using a LiF analyzing crystal and the scattered intensity measured with a $\mathrm{NaI}$ scintillation detector. This configuration resulted in an in-plane longitudinal resolution (i.e., resolution along the direction of $\mathbf{Q}$ in the scattering plane) of $\Delta Q_{\mathrm{L}}=0.005 \AA^{-1}$ full-width at half-maximum (FWHM). The instrumental resolution in the scattering plane and perpendicular to $Q$ was $\Delta Q_{\perp}=0.0005 \AA^{-1}$ FWHM, and the instrumental resolution out of the scattering plane was $\Delta Q_{\text {vert }}=0.042 \AA^{-1}$ FWHM. For the measurements described in this paper, which were generally made in the vicinity of an $(H K 0)$ peak, $\triangle Q_{\mathbf{L}}$ determines the radial resolution in the hexagonal basal plane, $\Delta Q_{\perp}$ determines the angular uncertainty corresponding to a rotation about the $Q_{z}$ axis, and $\Delta Q_{\text {vert }}$ determines the resolution normal to the $(H K 0)$ plane. Note that we use units $|\mathbf{Q}|=4 \pi \sin (\theta) / \lambda=2 \pi / d$.

The oven containing the strand was mounted in a standard 4-circle diffractometer. The geometry was such that the strand was approximately aligned along the axis of the $\phi$ circle. We define our angles such that $\phi=0$ and $\chi=0$ when the Bragg (100) peak is brought into the diffraction condition, as shown schematically in Figure 1. Thus, when $\chi=0$, a $\theta-2 \theta$ scan is equivalent to a radial scan with $\mathbf{Q}$ in the basal plane, and a scan in which $\phi$ alone varies results in $\mathbf{Q}$ tracing out a circle in the hexagonal basal plane. At a fixed value of $2 \theta$ and $\phi$, varying $\chi$ from 0 to $90^{\circ}$ brings $\mathbf{Q}$ from the basal plane to the $Q_{z}$, or $(00 L)$, axis.

Our strands typically consisted of a number of ordered domains with different orientations, similar to the "mosaic texture" of imperfect crystals. The mosaic texture of a strand was characterized by measuring the scattered X-ray intensity as a function of the $\phi$ and $\chi$ angles, at

fixed values of $2 \theta$ corresponding to the Bragg $\langle 100\rangle$ reflections, where $\left|Q_{\text {Bragg }}\right|=0.337 \AA^{-1}$. In most strands, several closely spaced domains in the strand could be identified (see for example Fig. 4a). Typical $\phi$ mosaic widths were $1-4^{\circ}$, although our best samples had widths that were less than $0.1^{\circ}$. Measurement of the $\chi$ mosaic was complicated by our poor instrumental resolution in this direction in reciprocal space, but our best samples had mosaics $\leq 6^{\circ}$. The maximum scattering at the peak of the Bragg $\{100\}$ peaks yielded a typical scattered intensity of $\sim 5 \times 10^{4}$ counts $/ \mathrm{s}$.

\section{Results of X-ray Measurements}

In reciprocal space, a toroid of diffuse scattering was found which enclosed the Bragg $\{100\}$ points (see Fig. 1). For the instrumental configuration utilized in these measurements, the intensity of this diffuse scattering was reduced by $\sim 4$ orders of magnitude from the elastic Bragg scattering. Initial measurements using photographic film indicated that the cross-section of the toroid was anisotropic, and extended farther along the $Q_{z}$ direction than in the basal plane. In the diffraction pattern shown in Figure 5, the sample was aligned so that the 100 Bragg peak satisfied the reflection condition; this peak is shown on one side of the beam trap. On the other side of the beam trap only diffuse scattering is seen. The $\phi$ mosaic in this ex- 


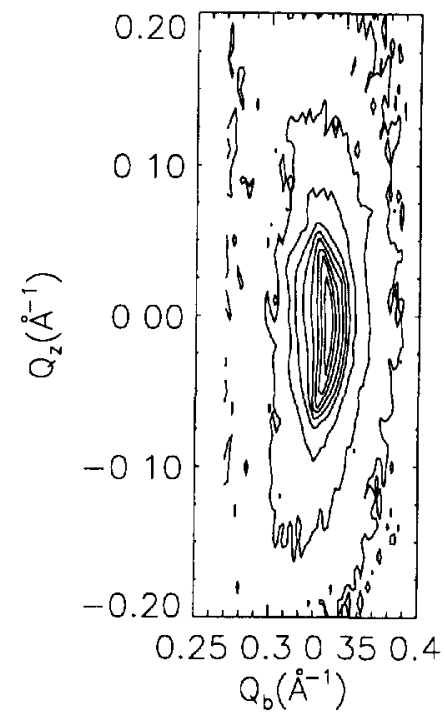

Fig 3. - Contour plot of the X-ray scattering intensity in a plane perpendicular to the basal plane and passing through a Bragg peak. Note the asymmetric extension of the diffuse scattering along the $Q_{z}$ direction as compared to the width of the scattering in the basal plane. The contours correspond to diffuse scattering intensities of $20,55,148,403,1097,2980,8103,22026$ and 59874 counts per $600 \mathrm{~s}$. This measurement was made with $\phi=0$, i.e., with the strand oriented so that the 100 peak was brought into the scattering condition.

periment was therefore less than $0.5^{\circ}$, and this scattering represents the cross section in the $H 0 L$ plane of the diffuse toroid. This cross section in reciprocal space is more or less elliptical. It is elongated along the $Q_{z}$, or column, axis, with dimensions about 4 times larger along $Q_{z}$ than perpendicular to it. Further quantitative measurements were done with the LiF analyzer configuration described above. A contour plot of the scattering in the $H O L$ plane at $\phi=\phi_{\text {Bragg }} \equiv 0$ is shown in Figure 3 . To quantitatively establish the cross-section of this toroid, measurements were made at fixed $Q_{z}$ while varying $\mathbf{Q}_{\mathrm{b}}$, and at fixed $\mathbf{Q}_{\mathrm{b}}$ while varying $Q_{z}$ (see for example Fig. 6). Here, $\mathbf{Q}_{\mathrm{b}}=Q_{x} \hat{\mathbf{x}}+Q_{y} \hat{\mathbf{y}}$, the projection of the $\mathbf{Q}$ vector on to the basal plane. In such measurements, it is important to avoid "contamination" from the much stronger elastic Bragg scattering. Thus, $\mathbf{Q}_{\mathrm{b}}$ scans were done at $Q_{z} \neq 0, Q_{z}$ scans were done at $\left|\mathbf{Q}_{\mathrm{b}}\right| \neq Q_{\mathrm{Bragg}}$, and both types of scan were done at $\phi \neq 0$. These measurements yield widths of $\Delta\left|\mathbf{Q}_{\mathrm{b}}\right|=0.10 \AA^{-1}$ FWHM and $\Delta Q_{z}=0.28 \AA^{-1}$ FWHM. Since $\Delta Q_{z}=0.28$ is much broader than the measured $Q_{z}$-width of the Bragg scattering (which is a convolution of the intrinsic mosaic and the instrumental resolution), and $\Delta\left|\mathbf{Q}_{\mathrm{b}}\right|=0.10$ is much broader than the measured $Q_{\mathrm{b}}$-width of the Bragg scattering, we conclude that these widths are intrinsic, and essentially unaffected by details of the resolution or sample mosaic. Although some models for the diffuse scattering $[10,11,13,14]$ predict divergences as $\mathbf{Q} \rightarrow \mathbf{Q}_{\mathrm{Bragg}}$, our relatively loose instrumental resolution, together with the relatively poor strand mosaic, did not allow us to make measurements extremely close to the Bragg peak, and measurements made far from the Bragg peak did not show any significant $\phi$ dependence of either the shape of the toroid cross-section or the magnitude of $|\mathbf{Q}|$ at the maximum. The fact that the radius of the diffuse scattering maximum was independent of $\phi$ indicated that the scattering lies in a circular ring in the $H O L$ plane, rather than in diffuse streaks connecting $\{100\}$ peaks as was previously 


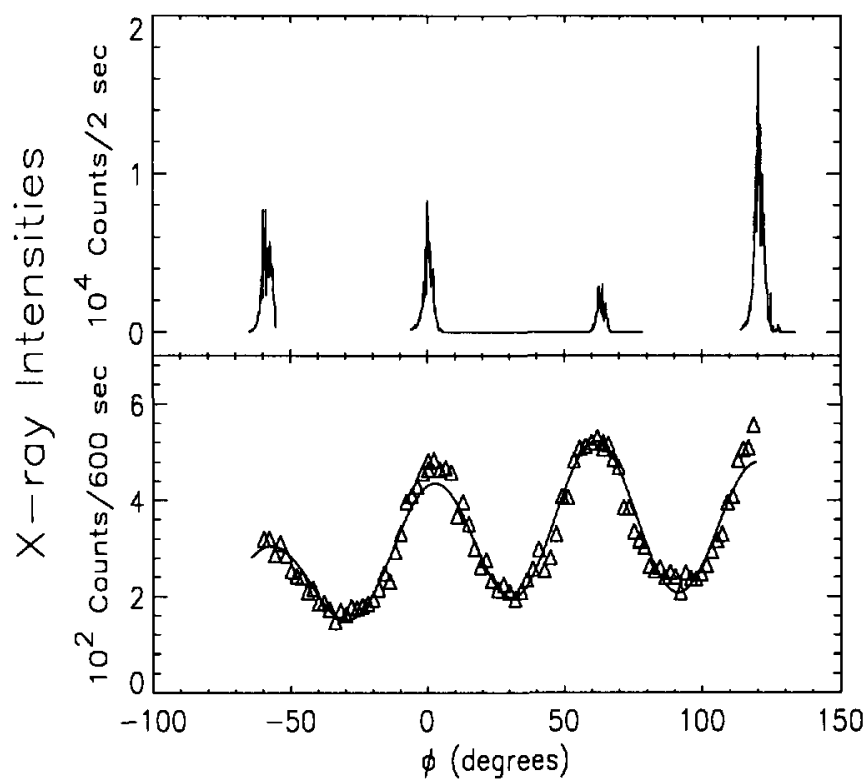

Fig. 4. - Top $\phi$-mosaic structure of a Bragg peak in a typical strand. The data collected from this strand correspond to data set 4 in Table I. The Bragg peak exhibits a FWHM of less than $4^{\circ}$, typical of the strands reported in thıs paper. The intensity variatıon of the Bragg $(100)$ peaks is due to a small misorientation of the strand axis with respect to the beam. Such a misalignment has a pronounced effect on the Bragg peak intensities, but has a negligible effect on measurements of the diffuse scattering intensity. Bottom: the $\phi$ dependence of the diffuse scattering in the same strand, at $\left|\mathbf{Q}_{\mathrm{b}}\right|=0.300$ and $L=000$. The maxima of the diffuse scattering occur at the $\phi$ angles of the Bragg $\langle 100\rangle$ peaks. The triangles indicate measured intensities as a function of $\phi$, while the solid curve shows the results of a least-squares fit to Equation (4).

observed in a different material [9].

Normally, one expects the intensity of the diffuse scattering associated with phonons or extended defects to decrease rapidly with increasing separation from a Bragg peak. To establish the shape of the diffuse scattering within the basal plane, further measurements of the diffuse scattering were made by fixing the length of $\mathbf{Q}$ and varying $\phi$. The diffuse scattering in three different strands was measured by fixing $\left|\mathbf{Q}_{\mathrm{b}}\right|=0.300 \AA^{-1}$ and $Q_{z}=0$, and varying $\phi$. (See, for example, Fig. 4b).) Note that in this case $|\mathbf{Q}|<\left|Q_{\text {Bragg }}\right|=0.337 \AA^{-1}$. Unlike the expected rapid decay away from $\phi=0\left(\bmod 60^{\circ}\right)$, these scans show a surprising sinusoidal variation in the intensity of the diffuse scattering around the ring. The peaks of the sinusoid are at the values of $\phi$ corresponding to the orientations of the Bragg points, and the minima correspond to the edges of the Brillouin zone. We emphasize that there is significant diffuse scattering at the edge of the Brillouin zone, well away from the Bragg peaks, even though the mosaic of the elastic scattering is only $\sim 1-4^{\circ}$. Additional measurements of the $\phi$ dependence were made on one of the above strands at $\left|\mathbf{Q}_{\mathrm{b}}\right|=0.370$ and $Q_{z}=0.100$ (i.e., $|\mathbf{Q}|=0.383>\left|Q_{\mathrm{Bragg}}\right|$ ), and a similar sinusoidal dependence was observed. 

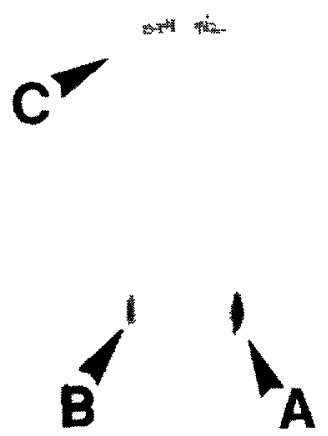

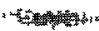

Fig. 5. - The strand axis is vertical in this photograph of the diffuse scattering from an aligned strand of HHTT. The exposure time for the photograph was four hours. "A" points to the strongly overexposed Bragg 100 reflection of the two-dimensional hexagonal lattice which satisfies the Bragg diffraction condition " $\mathrm{B}$ " points to the diffuse scattering in the basal plane away from the 100 Bragg reflection which is not oriented so as to satisfy the diffraction condition. " $\mathrm{C}$ " points to the 001 diffuse peak due to intracolumnar correlations (compare with Fig. 1). For this strand, the $\phi$ mosaic was measured to be $\Delta \phi \sim 0.25^{\circ} \ll \theta_{\text {Bragg }}$.
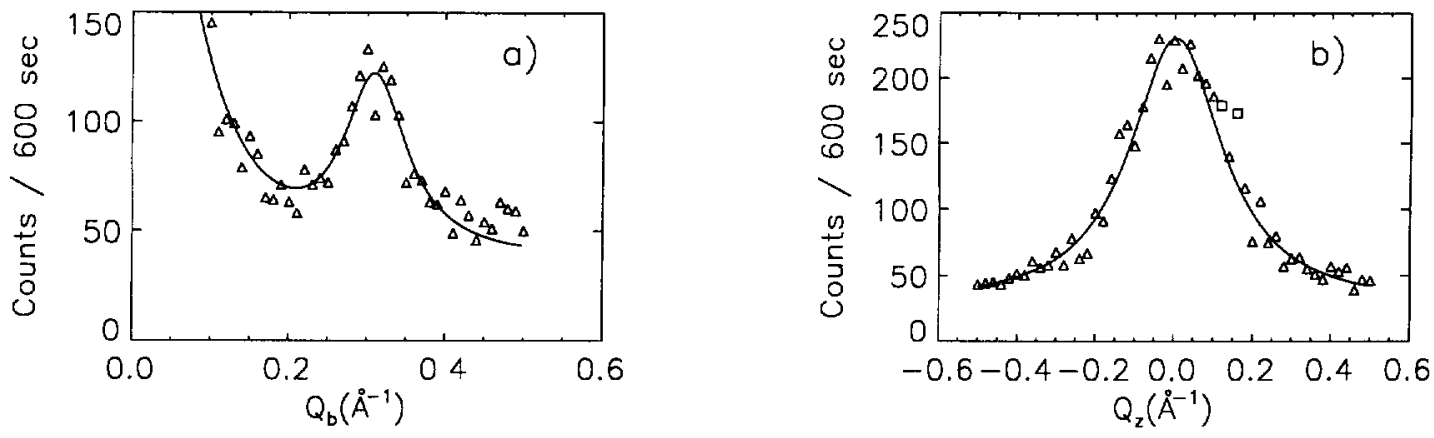

Fig. 6. - a) Triangles show the measured diffuse scattering intensity as a function of $\left|\mathbf{Q}_{\mathrm{b}}\right|$ with $\phi=15^{\circ}$ and $Q_{z}=0.10 \AA^{-1}$. The FWHM is $010 \AA^{-1}$. The smooth curve shows a fit to a Lorentzian peak together with an empirical background, determined by measuring the scattering from an empty cell and correcting for absorption by the sample. b) Triangles show the measured diffuse scattering as a function of $Q_{z}$ with $\phi=15^{\circ}$ and $Q_{\mathrm{b}}=0.300 \AA^{-1}$. The FWHM is $0.28 \AA^{-1}$. The smooth curve shows a Lorentzian fit with an empirically determined background as discussed above. Note that the two data points plotted as squares were attributed to Bragg contamination and were not included in the fit. 


\section{Analysis of $\phi$ Dependence of the Diffuse Scattering}

The observation of a sinusoidal variation of the diffuse scattering in the basal plane was unexpected. To further elucidate the nature of the sinusoidal modulation of the diffuse scattering and to establish the extent to which higher harmonics are present, the four data sets discussed above were analyzed via least squares fits to a sinusoidal function. The simplest model function would be a constant plus a simple sine wave. For our initial fits we used the following function:

$$
I_{\text {calc }}=b+c \mathcal{L}(\phi)+d(1+e \mathcal{L}(\phi)) \sin \left(6 \phi+\delta_{1}\right)
$$

where

$$
\mathcal{L}(\phi)=\sin (2 \phi+\gamma) .
$$

The $\mathcal{L}(\phi)$ term accounts for a slow variation in both the sinusoidal term and the "background" which we attribute to a small misalignment of the apparatus. If the strand is displaced from the center of rotation of the goniometer by even $\sim 30-50 \mu$ it can "wobble" to a significant extent in the X-ray beam as $\phi$ is varied, resulting in a sub-harmonic with period $180^{\circ}$ in $\phi$ and a phase $\gamma$ depending on the direction of misalignment. As can be seen in Fig. $4 \mathrm{~b}$, the fit to the function in Equation (2) is remarkably good. Similarly good fits are found comparing the simple sinusoidal function to the other four data sets.

Clearly, any function describing the variation of the diffuse scattering in the hexagonal basal plane should mirror the lattice symmetry, i.e., 6-fold rotational symmetry plus inversion symmetry. Any such function can be decomposed into a Fourier series, $\sum_{n} C_{n} \sin \left(6 n \phi+\delta_{n}\right)$. The convention $\phi_{\mathrm{Bragg}}=0$ defines $\delta_{1}=0$. The inversion symmetry of the hexagonal lattice then implies that $\delta_{n}=\delta_{1}=0$ for all $n>1$, with $C_{n}$ free to be either positive or negative. Our initial analysis showed that truncating the sernes at $n=1$ already provided a good fit to the data. The extent to which higher harmonics contribute remained to be determined. Accordingly, we fitted our data to a function:

$$
I_{\text {calc }}=b+c \mathcal{L}(\phi)+(1+e \mathcal{L}(\phi)) \sum_{n=1}^{4} C_{n} \sin \left(6 n \phi+\delta_{n}\right)
$$

with $\mathcal{L}(\phi)$ defined as in Equation (3). For most fits we allowed $C_{1}$ and at most one other $C_{n}$ to be nonzero, and fixed $\delta_{n}=\delta_{1}$ for $n>1$. $\delta_{1}$ was determined by the strand orientation. Though allowing a free phase for the higher harmonics always significantly improves the goodness-of-fit, there is no physical basis for the development of an arbitrary phase between the fundamental and a harmonic. (A phase shift of $\pi$ can be accounted for by a negative value for $C_{n}$ ). We used Hamilton's test [28] to establish whether the addition of the $n^{\text {th }}$ harmonic term improves the quality of the fit in a statistically significant manner. This is accomplished through a comparison of the model containing only the fundamental, to the model containing the $n^{\text {th }}$ harmonic in addition to the fundamental. The former model, found in Equation (2) has one less fitted parameter than the latter model in Equation (4) with one other $C_{n}$ allowed to be nonzero.

Using Hamilton's test at the $90 \%$ confidence level to compare the simple model of Equation (2) to the $n^{\text {th }}$ harmonic model, for $n=2,3$, and 4 , we found that the $n=2$ harmonic is statistically significant in one of the four data sets, while the $n=3$ term is significant in one and the $n=4$ term is significant in one of the four data sets (see Tab. I). If the $n=2$ harmonic in Equation (4) is allowed to have a variable phase, the resultant goodness-of-fit improves greatly. In this case, Hamilton's test at the $90 \%$ confidence level would confirm the existence of the $n=2$ harmonic in all four of the data sets. However, as discussed above 
Table I. - Parameters found in least-squares fits to a sinusordal function for the $\phi$-dependent diffuse scattering, as described in text. The second and third columns give the values of $\left|\mathbf{Q}_{\mathrm{b}}\right|$ and $Q_{z}$ at which the measurement was made. The fourth, fifth, and sixth columns gives values of $C_{2} / C_{1}, C_{3} / C_{1}$, and $C_{4} / C_{1}$ from fits to Equation (4). Statistıcally significant parameters are given with error bars. Parameters that are not statıstically significant are given in parentheses. Note that the statistically significant parameters have uncertainty ranges which come close to including zero [12].

\begin{tabular}{|c|c|c|c|c|c|}
\hline Data Set & $\left|\mathrm{Q}_{\mathrm{b}}\right|\left(\AA^{-1}\right)$ & $Q_{z}\left(\AA^{-1}\right)$ & $C_{2} / C_{1}$ & $C_{3} / C_{1}$ & $C_{4} / C_{1}$ \\
\hline 1 & 0.300 & 0.00 & $-0.154 \pm 0.152$ & $(0.029)$ & $(-0.013)$ \\
2 & 0300 & 0.00 & $(0.007)$ & $(0013)$ & $(-0.004)$ \\
3 & 0.300 & 0.00 & $(0.023)$ & $0.113 \pm 0.112$ & $0.060 \pm 0.047$ \\
4 & 0.370 & 0.10 & $(-0.013)$ & $(0.011)$ & $(-0.021)$ \\
\hline
\end{tabular}

we believe that the inclusion of additional free phases is unphysical. Thus, we attribute the appearance of an "offset" $n=2$ harmonic to astatistical noise in our measurement, perhaps arising from small misaligned domains in the strand.

As a final application of Hamilton's test, the uncertainties in the amplitudes of the $n=$ 2 harmonic are explored. The same Hamilton's test comparison is made using the fitted parameters in the model with one higher harmonic. By fixing the amplitude of the higher harmonic at values successively farther from the ideal fitted value, we can determine what higher harmonic amplitude causes the $\mathcal{R}$ of Hamilton's test to cross the one-standard-deviation level, where $\mathcal{R}=R_{1} / R_{0}$ and $R_{0}$ and $R_{1}$ are respectively the generalized weighted $R$ factors for a structure resulting from an unrestrained least-squares refinement and a refinement with restraints on some of the parameters [28]. In this manner, the uncertainty in the amplitude of the higher harmonic can be determined by our fits to the data. The results of our analysis using Hamilton's test are summarized in Table I.

In summary, we were able to obtain excellent fits to the basal plane intensity variation of the diffuse scattering, by using only a simple sinusoidal model. In most of our measurements, we found that the $n=2,3$, and 4 harmonics are statistically insignificant. Even in data sets where such harmonics could be statistically justified, the uncertainty in the amplitude of the harmonic brings the amplitude very close to null.

\section{X-ray Diffuse Scattering by Elastic Waves in the Hexagonal Phase}

The anisotropy of the X-ray diffuse scattering in the vicinity of a Bragg reflection can be related to the elastic properties of the columnar phase. In the following discussion, we will consider the role played by phonons and we calculate their contribution to the diffuse scattering. However, there are other explanations which a prior apply as well as the model of phonons presented here. For instance, the diffuse scattering may arise from static defects: either dislocations as proposed by Bouligand [13] or from point defects and lock-in faults as proposed by Prost [14]. The scattering by such defects is then related to the displacement field around their cores [7]. This field is governed by the elastic energy of the medium in the same way that the phonon energy and dispersion are. In both cases, the shape of the diffuse scattering appears to be driven mainly by the values of the different elastic constants. Therefore, one can predict that comparable diffuse scattering shapes should be observed in the case of phonons and of 
static defects, at least as far as the anisotropy in the $H 0 L$ plane is concerned [29]. A possible way to discriminate between these two models would be to perform an energy analysis of the scattering, for example via neutron scattering. The small monodomain size achieved to date seriously restricts the feasibility of such an experiment.

The elastic energy density of a hexagonal columnar phase has been derived by several authors $[10,14,30]$. This energy contains two contributions: that of a two-dimensional hexagonal solid medium, and a Frank liquid-crystalline energy, containing the three usual terms: the splay (elastic constant $k_{1}$ ), twist (elastic constant $k_{2}$ ), and bend (elastic constant $k_{3}$ ) contributions [31]. Following [30], the elastic energy density, $f$, is given by

$$
\begin{aligned}
f= & \frac{1}{2}(\lambda+2 \mu)\left(\left(\frac{\partial u}{\partial x}\right)^{2}+\left(\frac{\partial v}{\partial y}\right)^{2}\right)+\lambda \frac{\partial u}{\partial x} \frac{\partial v}{\partial y}+\frac{1}{2} \mu\left(\frac{\partial u}{\partial y}+\frac{\partial v}{\partial x}\right)^{2} \\
& +\frac{1}{2} k_{3}\left(\left(\frac{\partial^{2} u}{\partial z^{2}}\right)^{2}+\left(\frac{\partial^{2} v}{\partial z^{2}}\right)^{2}\right)^{2}
\end{aligned}
$$

where $u$ and $v$ are the components of the displacement vector $\mathbf{u}$, which is perpendicular to the director $\mathbf{n}$ of the hexagonal phase. We assume that $\mathbf{n}$ varies slightly from the fixed $\hat{\mathbf{z}}$ direction:

$$
\begin{aligned}
\mathbf{u}(x, y, z) & =u(x, y, z) \hat{\mathbf{x}}+v(x, y, z) \hat{\mathbf{y}} \\
\mathbf{n}(x, y, z) & =\hat{\mathbf{z}}+\frac{\partial \mathbf{u}}{\partial z}
\end{aligned}
$$

$\lambda$ and $\mu$ are the Lamé elastic constants of a two-dimensional hexagonal solid. (The sixfold symmetry induces isotropic two-dimensional elastic properties). Only the bend contribution (elastic constant $k_{3}$ ) is included in $f$. As shown in [32], the two other terms are forbidden in a so-called developable domain of a columnar medium. In [10], a complete derivation including the splay (elastic constant $k_{1}$ ) and twist (elastic constant $k_{2}$ ) terms is presented, and we will compare our results with this derivation.

From Equation (5), one derives the two propagation equations for an elastic wave of displacement $\mathbf{u}$, which can be collected in a single vectorial equation:

$$
(\lambda+\mu) \nabla_{\perp}\left(\nabla_{\perp} \cdot \mathbf{u}\right)+\mu \nabla_{\perp} \mathbf{u}-k_{3} \frac{\partial^{4} \mathbf{u}}{\partial z^{4}}=\rho \frac{\partial^{2} \mathbf{u}}{\partial t^{2}}
$$

Equation (8) is solved in Fourier space, writing $\mathbf{u}=\mathbf{u}_{0} \mathrm{e}^{2(\mathbf{q} \mathbf{r}-\omega t)}$, where $\mathbf{q}=\mathbf{q}_{\mathrm{b}}+q_{z} \hat{\mathbf{z}}$ is the wave vector of one phonon mode. Let us choose the $\hat{\mathbf{x}}$ direction along $\mathbf{q}_{\mathrm{b}}$ and call $\theta$ the angle between $\mathbf{q}_{\mathrm{b}}$ and $\mathbf{u}_{0}: \mathbf{q}_{\mathrm{b}}=q_{\mathrm{b}} \hat{\mathbf{x}}$ and $\mathbf{u}_{0}=u_{0} \cos (\theta) \hat{\mathbf{x}}+u_{0} \sin (\theta) \hat{\mathbf{y}}$. Projections on the $\hat{\mathbf{x}}$ and $\hat{\mathbf{y}}$ axes read:

$$
\begin{aligned}
& \cos (\theta)\left((\lambda+2 \mu) q_{\mathrm{b}}^{2}+k_{3} q_{z}^{4}-\rho \omega^{2}\right)=0 \\
& \sin (\theta)\left(\mu q_{\mathrm{b}}^{2}+k_{3} q_{z}^{4}-\rho \omega^{2}\right)=0
\end{aligned}
$$

This system has two solutions, for $\theta=0$ and $\theta=\pi / 2$ :

$$
\begin{array}{ll}
\text { mode 1: } & \theta=\frac{\pi}{2} \Rightarrow \mathrm{u}_{01} \perp \mathrm{q}_{\mathrm{b}} \text { and } \rho \omega_{1}^{2}=\mu q_{\mathrm{b}}^{2}+k_{3} q_{z}^{4} \\
\text { mode 2: } & \theta=0 \Rightarrow \mathbf{u}_{02} \| \mathbf{q}_{\mathrm{b}} \text { and } \rho \omega_{2}^{2}=(\lambda+2 \mu) q_{\mathrm{b}}^{2}+k_{3} q_{z}^{4}
\end{array}
$$

Let us calculate the contribution of these two modes to the diffuse scattering around a Bragg reflection of reciprocal vector $\mathbf{Q}_{\mathrm{Bragg}}$. We call $I(\mathbf{Q})$ the scattered intensity at the vector $\mathbf{Q}=\mathbf{Q}_{\mathrm{Bragg}}+\mathbf{q}$, where $\mathbf{q}$ is the phonon wave vector. We define another coordinate system, $\hat{\mathbf{X}}$, 


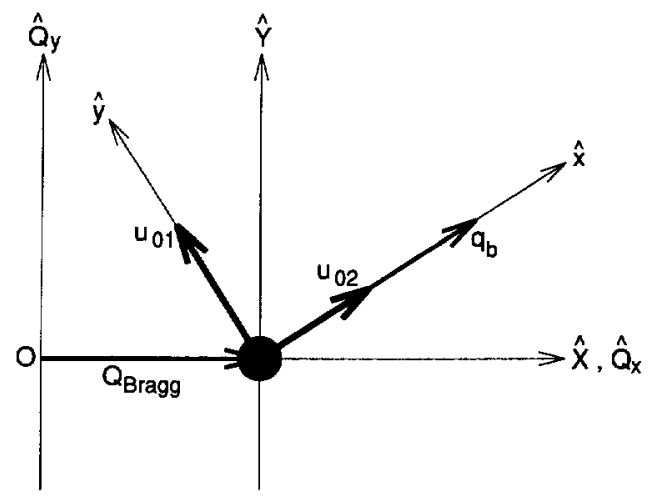

Fig. 7. - Conventions used in the analysis of Section 4. The phonon wave vector is referred to as $q$ in this paper, and has its origin at a Bragg point, $\mathbf{Q}_{\mathrm{Bragg}}$. Only the basal plane is shown in the Figure: $\mathrm{q}_{\mathrm{b}}$ refers to the projection of the phonon wave vector on to the basal plane. A first set of axes, $\hat{\mathbf{x}}, \hat{\mathbf{y}}$ is defined at a Bragg point with $\hat{\mathbf{x}}$ along the $q_{b}$ direction and $\hat{\mathbf{y}}$ perpendicular to $\hat{\mathbf{x}}$. A second set of axes, $\hat{\mathbf{X}}, \hat{\mathbf{Y}}$, is also defined at the Bragg point with $\hat{\mathbf{X}}$ along the $\mathbf{Q}_{\mathbf{B r a g g}}$ direction and $\hat{\mathbf{Y}}$ perpendicular to $\hat{\mathbf{X}}$.

$\hat{\mathbf{Y}}$ (origin at the vector $\mathbf{Q}_{\mathbf{B r a g g}}$ and $\hat{\mathbf{X}}$ along the $\mathbf{Q}_{\mathbf{B r a g g}}$ direction) as shown in Figure 7. For small displacement amplitudes [33], $I(\mathbf{Q})$ is proportional to:

$$
I(\mathbf{Q}) \propto\left(\mathbf{u}_{01} \cdot \mathbf{Q}\right)^{2}+\left(\mathbf{u}_{02} \cdot \mathbf{Q}\right)^{2}
$$

The equipartition theorem gives $\left|\mathbf{u}_{01}\right|^{2} \propto k_{\mathrm{B}} T / \omega_{1}^{2}$, and, remembering the polarization directions of the two modes given above, one has:

$$
\begin{aligned}
I(Q) & \propto \frac{1}{q_{X}^{2}+q_{Y}^{2}}\left(\frac{Q_{\mathrm{Bragg}}^{2} q_{Y}^{2}}{\omega_{1}^{2}}+\frac{1}{\omega_{2}^{2}}\left(Q_{\mathrm{Bragg}} q_{X}+q_{X}^{2}+q_{Y}^{2}\right)^{2}\right) \\
\omega_{1}^{2} & \propto \mu\left(q_{X}^{2}+q_{Y}^{2}\right)+k_{3} q_{Z}^{4} \\
\omega_{2}^{2} & \propto(\lambda+2 \mu)\left(q_{X}^{2}+q_{Y}^{2}\right)+k_{3} q_{Z}^{4}
\end{aligned}
$$

We introduce the length $\Lambda=\sqrt{k_{3} /(\lambda+2 \mu)}$ and the ratio $\alpha=(\lambda+2 \mu) / \mu$ (with $\left.2 \leq \alpha \leq+\infty\right)$, $I(\mathbf{Q})$ finally reads

$$
\begin{aligned}
& I(\mathbf{Q}) \propto I\left(\mathbf{Q}_{\mathrm{Bragg}}+\mathbf{q}\right) \\
& \propto \frac{1}{\left(q_{X}\right)^{2}+\left(q_{Y}\right)^{2}}\left(\frac{\alpha\left(Q_{\mathrm{Bragg}} q_{Y}\right)^{2}}{\left(q_{X}\right)^{2}+\left(q_{Y}\right)^{2}+\alpha \Lambda^{2}\left(q_{Z}\right)^{4}}+\frac{\left(Q_{\mathrm{Bragg}} q_{X}+\left(q_{X}\right)^{2}+\left(q_{Y}\right)^{2}\right)^{2}}{\left(q_{X}\right)^{2}+\left(q_{Y}\right)^{2}+\Lambda^{2}\left(q_{Z}\right)^{4}}\right)
\end{aligned}
$$

As expected, Equation (16) is equivalent to the comparable expression of [10] with $k_{1}$ and $k_{2}$ set to zero. In [10], the exponential Debye-Waller term is taken into account, but one can neglect its influence in our range of interest, that is, in the vicinity of a single Bragg reflection.

\section{Discussion}

To compare our measurements with Equation (16), we need to determine the two parameters $\Lambda$ and $\alpha$. This is accomplished in two successive steps: first, we adjust $\Lambda$ to agree with the data in the $H 0 L$ plane, since only $\Lambda$ governs the scattered intensity in this plane. To reproduce 


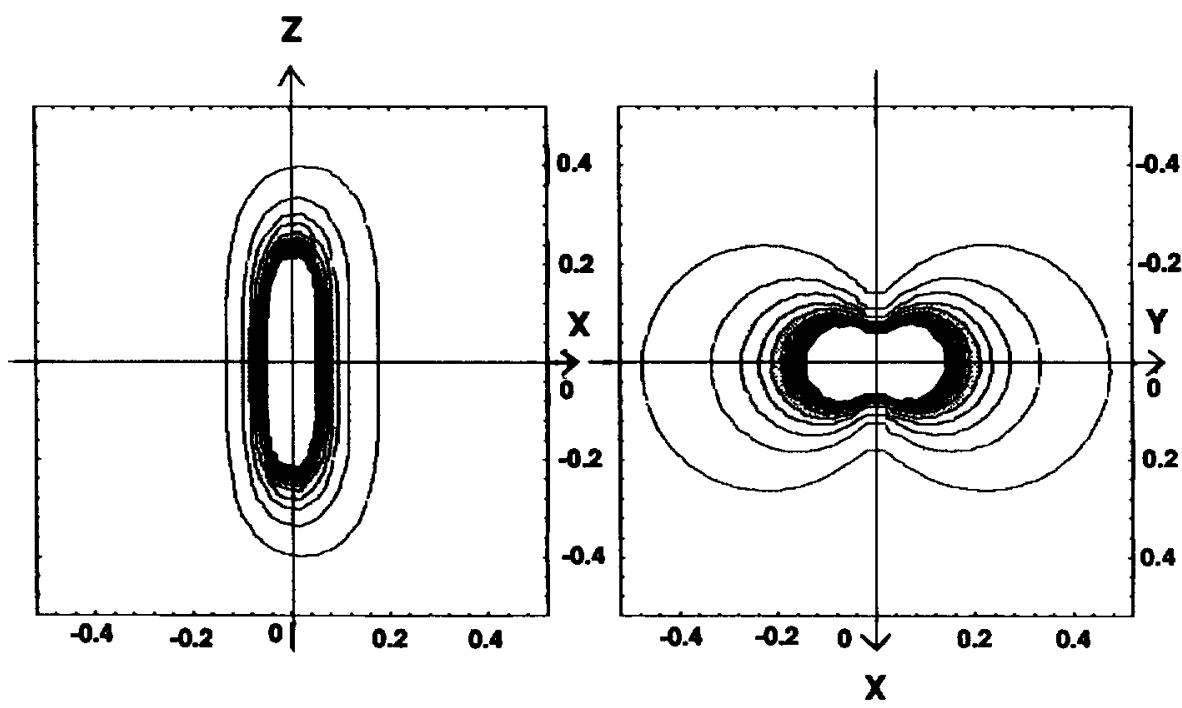

Fig. 8. - Isointensity contours for calculated diffuse scattering $I(\mathbf{Q})$ from phonons (from Eq. (16)), drawn in the reciprocal space $\hat{\mathbf{X}} \hat{\mathbf{Z}}$ and $\hat{\mathbf{X}} \hat{\mathbf{Y}}$ planes about the (100) reflection (see Fig. 7 ). We show 10 contours equally spaced in intensity between 0 and 500 (arbitrary units). Axıs units are normalızed to $\left|\mathbf{Q}_{\mathrm{Bragg}}\right|=\mathbf{0 . 3 3 4} \AA^{-1}$. Only the contribution arising from one Bragg peak is shown. For the plots shown we chose $\Lambda=1.5 \AA$ and $\alpha=10$.

the observed anisotropy of the scattering, we found that it was necessary to choose $\Lambda$ in the range $1.5 \AA \leq \Lambda \leq 4 \AA$. Then, $\Lambda$ being fixed in this range, we adjust $\alpha$ to optimize agreement with the data in the $H K O$ plane. A typical result is shown in Figure 8. Such a value for $\Lambda=\sqrt{k_{3} /(\lambda+2 \mu)}$ should be compared with previous mechanical measurements reported in the literature. Although there are very few precise measurements of the elastic constants of discotics, from measurement in discotic nematics [34,35] and $D_{h}$ phases [36] we can estimate $k_{3} \approx 1-4 \times 10^{-12} \mathrm{~N}$ and $B \sim 10^{8} \mathrm{~m}^{2} / \mathrm{N}[37,38]$. This then gives $\Lambda=\left(\mathrm{k}_{3} / \mathrm{B}\right)^{1 / 2} \approx 1-3 \AA$, in good agreement with our fitted value. This rather small value of $\Lambda$ implies that the columns bend more easily than they compress, which is demonstrated by an analogy with the smectic A phase, following de Gennes [31]. We can relate $\Lambda$ to a penetration length $L$, which has more physical significance. A small undulation of the cylinders at some interface induces a distorted region of thickness $L$. Assuming a wavelength of $2 \pi / k$ for the undulation gives $L=1 / k^{2} \Lambda$. Taking $\Lambda=2 \AA$ and $2 \pi / k=10 \mu \mathrm{m}$, one finds $L=13 \mathrm{~mm}$. (By contrast, in smectic phases, one typically finds $L=1 \mathrm{~mm}$ [31]). This indicates that, in a 50-200 $\mu \mathrm{m}$ diameter strand, surface effects may play an important role. Thus, the phonon model describes the $H 0 L$ scattering well, although, as discussed above, models for topological or other static defects might also be expected to provide a good description of the data.

The phonon model provides a less satisfactory agreement with the $\phi$ dependence of the diffuse scattering, i.e. the lineshape in the $H K O$ plane. As discussed in Sections 3 and 4, scans in which $\phi$ was varied, at different values of $H$ and $L$ and fixed $\left|\mathbf{Q}_{\mathrm{Bragg}}\right|$, indicated a smooth sixfold sinusoidal dependence of the scattering, with little or no contribution from higher harmonics. The analysis presented in Section 5 predicts a divergence as $\mathbf{Q} \rightarrow \mathbf{Q}_{\mathrm{Bragg}}$, which of course would imply an infinite set of harmonics, but also does not agree with the measured $\phi$ dependence at other values of $|\mathbf{Q}|$. To use the phonon model in the $H K 0$ plane, 
the value of $\alpha$ has to be large enough to reproduce the slow decay away from the Bragg reflection of the diffuse scattering versus $\phi$ (see Fig. 4). However, for such large values of $\alpha$, Equation (16) actually predicts two poorly resolved maxima in the scattered intensity at $\pm \Delta \phi$, while the measured intensity shows a single maximum at $\phi=0$. This rather strange effect is due to the detailed shape of the scattering in the $H K 0$ plane predicted by the model (see the right-hand side of Fig. 8) and is only predicted for scans made at $\mathbf{Q} \neq \mathbf{Q}_{\mathrm{Bragg}}$. Since this effect is not experimentally observed, we have to restrict the value of $\alpha$ to the range $2 \leq \alpha \leq 10$. In summary, the phonon model does not provide satisfactory quantitative agreement with the observed diffuse scattering in the $H K 0$ plane.

Indeed, the $\phi$ dependent sinusoidal variation in the intensity of the diffuse scattering is not predicted by any publsshed theory that we are aware of. It should be noted that a calculation of the scattering from phonons or static defects, such as that presented in Section 5, generally relies on a small $\Delta Q$ approximation. Thus, most of the scattering that we have measured is outside the regime of validity of such theories. Indeed, the observation of substantial diffuse scattering all the way out to the zone boundary is surprising in itself. Qualitatively speaking in terms of phonons, this observation implies that transverse phonons (2D shear deformations involving only the constant $\mu$ ) of all wavelengths cost little energy compared to that of longitudinal phonons and also to $k_{\mathrm{B}} T$. If the scattering is considered to arise from static defects rather than dynamic fluctuations, this observation implies that the defects must be highly localized. Also, the relatively small radial extension of the scattering about the Bragg reflections in the $H K O$ plane indicates that the defects do not involve strong density fluctuations.

The question still remains open as to why the diffuse scattering of hexapentyloxytriphenylene [9] consists of diffuse sheets whereas that of HHTT has a toroidal shape. Preliminary qualitative experiments (using photographic plates) on phasmidic compounds [39] and on lyotropic hexagonal phases [11] have also shown a similar toroidal shape. In the case of hexapentyloxy triphenylene [9], the diffuse scattering may arise from the existence of screw dislocation lines oriented along the $\langle 100\rangle$ directions [11].

Further calculations are clearly required in order to elucidate the source of the sinusoidal intensity modulation around the toroid of diffuse scattering in HHTT and related materials.

\section{Acknowledgments}

We are grateful to M. Cagnon, E. Dubois-Violette, G. Durand, A. M. Levelut, T. C. Lubensky, B. Pansu and J. Prost for useful discussions. Work at the University of Pennsylvania was supported by National Science Foundation Grants DMR MRL 92-20668 and DMR 93-15341.

\section{References}

[1] See, e.g., Chandrasekhar S. and Ranganath G. S., Rep. Prog Phys 53 (1990) 57 for a recent review.

[2] Chandrasekhar S., Sadashiva B. K. and Suresh K., Pramana 9 (1977) 471.

[3] Levelut A. M., J. Chım Phys. 80 (1983) 149.

[4] Frank F. C. and Chandrasekhar S. J ,J. Phys. France 41 (1980) 1285.

[5] Levelut A. M. , Oswald P., Ghanem A. and Malthete J , J. Phys. France 45 (1984) 745.

[6] Pershan P. S., Structure of Liquid Crystal Phases (World Scientific Pub. Co., Singapore, 1988) and references therein 
[7] Davidson P., Pansu B , Levelut A. M. and Strzelecki L., J. Phys. II France 1 (1991) 61.

[8] Fontes E., Heiney P. A., Ohba M, Haseltine J. N. and Smith, III A B, Phys Rev. A 37 (1988) 1329.

[9] A. M. Levelut, J. Phys Lett. France 40 (1979) L81.

[10] Selinger J. V. and Bruinsma R. F., Phys. Rev. A 43 (1991) 2910.

[11] Clerc M., Thesıs, Université Paris-Sud, Centre d'Orsay (1992).

[12] In addition to the measurements of the $\phi$ dependence of the diffuse scattering intensity presented in this paper, a fifth data set was discarded due to the poor quality of the Bragg mosaic.

[13] Bouligand Y., J. Phys. France 41 (1980) 1297.

[14] Prost J., Lıq Cryst. 8 (1990) 123.

[15] Selinger J V and Bruinsma R. F., J. Phys. II France 2 (1992) 1215

[16] Heiney P. A. and Ghosh S. S., unpublished.

[17] Fontes E, Heiney P. A. and de Jeu W. H., Phys. Rev. Lett 61 (1988) 1202.

[18] Heiney P. A., Fontes E., de Jeu W. H., Riera A, Carroll P. and Smith, III A B., J. Phys. France 50 (1989) 461.

[19] Fontes E., Ph.D. Thesis, University of Pennsylvania (1989) unpublished.

[20] Idziak S. H. J., Heıney P. A, McCauley, Jr. J. P., Carroll P. and Smith, III A. B., Mol. Cryst. Liq Cryst Lett. 237 (1993) 271.

[21] Kohne B., Poules W. and Praefcke K., Chem. Ztg. 108 (1983) 113.

[22] Praefcke K., Kohne B., Poules W. and Poetsch E., (Merck Patent B.m.b.H.) Ger. Offen. DE 3, 346,980 (Cl. C07C149/36) 04 July, 1985.

[23] Lee W. K., Heiney P. A., McCauley, Jr. J. P. and Smith, III A. B., Mol. Cryst Lıq. Cryst. 198 (1991) 273.

[24] Van Winkle D. H and Clark N A., Phys. Rev. Lett. 48 (1982) 1407.

[25] Safinya C. R., Liang K. S., Varady W. A., Clark N. A. and Anderssen G., Phys. Rev. Lett. 53 (1984) 1172.

[26] Details of the strand apparatus are described in detail in references $[8,18,19]$ The strand oven was filled with helium to avord sample oxidation. The material was placed in a cup and heated into the isotropic phase. A pin was then inserted into the cup, and the material was cooled into its mesophase. Finally, the pin was pulled up slowly to grow a strand of discotic liquid crystal $\sim 2.5 \mathrm{~mm}$ in height and $150-200 \mu \mathrm{m}$ in diameter.

[27] Details of the X-ray diffraction apparatus for these measurements have been described in: Degert C., Davidson P., Megtert S., Petermann D. and Mauzac M., Lıq. Cryst. 12 (1992) 779. For these measurements, the wavelength was $1.541 \AA$ and the sample-film distance was $70 \mathrm{~mm}$.

[28] Hamilton W. C., Acta Cryst. 18 (1965) 502.

[29] Prost J., Private communication.

[30] Oswald P. and Kleman M., J. Phys. France 42 (1981) 1461.

[31] de Gennes P. G., The Physics of Liquid Crystals (Oxford University Press, London, 1974).

[32] Kleman M., J. Phys France 41 (1980) 737.

[33] Guinier A., X-ray diffraction in crystals, imperfect crystals, and amorphous bodies (W. H. Friedmann and Co., San Francisco, 1963).

[34] Heppke G., Kneppe H., Quentel S., Ranft A. and Sabaschus B., Mol. Cryst. Lıq. Cryst Lett. 81 (1991) 17.

[35] Warmerdam T. W., Frenkel D. and Zijlstra R J. J , Lıq Cryst. 3 (1988) 369.

[36] Oswald P., J. Phys. Lett. France 42 (1981) L171.

[37] Gharbia M., Cagnon M. and Durand G., J. Phys Lett. France 46 (1985) L683.

[38] Gharbia M, Othman T., Gharbi A., Destrade C and Durand G, Phys. Rev. Lett. 68 (1992) 2031

[39] Malthete J. and Davidson P., Bull. Soc. Chrm France, to be published. 